\title{
LA ORIENTACIÓN METACOGNITIVA Y EL DESARROLLO DE LA AUTOESTIMA: EVALUACIÓN DEL «PROGRAMA DE ESTRATEGIAS METACOGNITIVAS PARA EL DESARROLLO HUMANO»
}

\author{
METACOGNITIVE GUIDANCE AND DEVELOPMENT OF SELF-ESTEEM: \\ EVALUATION OF THE «PROGRAM OF HUMAN DEVELOPMENT THROUGH \\ METACOGNITIVE STRATEGIES»
}

\author{
Elvira Repetto* y Raúl Carvallo**
}

UNED y Universidad Veracruzana

\begin{abstract}
RESUMEN
Los autores exponen la posibilidad del desarrollo humano de alumnos universitarios a través de un programa de estrategias metacognitivas y su incidencia en el incremento de la autoestima. Se discute, primero, los conceptos de desarrollo humano y de autoestima así como los de estrategias metacognitivas, revisando las teorías más relevantes desde las psicosociales, las de la cognición social, el desarrollo de sí mismo, los factores asociados a la autoestima y la autorregulación de los procesos cognitivos. A continuación, y partiendo de un análisis de necesidades, se sintetizan los elementos del «Programa de Estrategias Metacognitivas para el Desarrollo Humano». La parte central del estudio presenta la evaluación del programa, tanto la inicial y su viabilidad como la del proceso y sus resultados. Por último, se recogen las conclusiones e implicaciones del estudio para futuras investigaciones.
\end{abstract}

Palabras clave: Autoestima, autoeficacia, autodignidad, desarrollo humano, crecimiento personal, enseñar a pensar, estrategias metacognitivas, programa de orientación metacognitiva.

\section{ABSTRACT}

The authors posit that human development of higher education students is possible through a program of metacognitive strategies and discuss its incidence on the increase of self-esteem. They present the

* Elvira Repetto Catedrática de Orientación Educativa. Es Presidenta de la AEOP y Corresponsal Nacional de la AIOSP. Entre sus líneas de investigación está la Metacognición y la Comprensión Lectora, la Formación y las Competencias a nivel internacional y el desarrollo de la Carrera. Ha dirigido varias tesis doctorales en estos temas.

** Raúl Carvallo. Profesor de la Universidad de Veracruz- México. Ha realizado la tesis doctoral en el ámbito de la Metacognición y trabaja en el área del desarrollo humano y la metacognición. 
concepts of human development and self-esteem, as well as metacognitive strategies, supporting them with the most relevant theories underpinning these concepts. After a needs assessment, they synthesize the elements of the «Program of Human Development through Metacognitive Strategies». The central part of the study focuses on the evaluation (initial, process, and results) and viability of the program. Finally, conclusions and implications for practice and for future studies are presented.

Key words: Self-esteem, self-dignity, human development, personal growth, learning strategies, metacognitive guidance.

\section{Introducción}

En los últimos años se han realizado esfuerzos para dar respuesta a los problemas de aprendizaje que tienen los alumnos. Actualmente se ha atribuido una mayor importancia a aquellos objetivos relacionados con la enseñanza de procesos y estrategias, así como al desarrollo de programas específicos para enseñar a pensar. En un nivel, generalmente independiente, se han promovido estudios sobre los aspectos motivacionales relacionados con el aprendizaje sin llegar a integrarse formalmente en los estudios sobre el desarrollo del pensamiento y el desarrollo afectivo o emocional de los estudiantes. Este tercer supuesto es precisamente el que ha motivado el presente estudio sobre la incidencia de las estrategias metacognitivas en el desarrollo personal de los alumnos.

En concreto, el presente artículo recoge los aspectos más relevantes de la investigación realizada por los autores que pretenden valorar la eficacia de la aplicación del «Programa de Estrategias Metacognitivas para el Desarrollo Humano» (Carvallo, 1999) con alumnos universitarios del primer curso de la Facultad de Psicología de la Universidad Veracruzana (México).

\section{Fundamentación teórica}

En las últimas décadas se han realizado diversos estudios para dar respuesta a los problemas de aprendizaje de los alumnos, en especial, se han elaborado y aplicado programas para enseñar a pensar. Asímismo, son numerosos los estudios sobre aspectos motivacionales del alumnado y su desarrollo emocional. Pero apenas se hallan en la literatura especializada trabajos que estudien la incidencia de las estrategias metacognitvas en el desarrollo personal de los alumnos de nivel universitario, que ya poseen preconcepciones sobre sí mismos.

Por ello, el objetivo del presente trabajo es la evaluación de un Programa para el desarrollo humano a través de estrategias metacognitivas en los alumnos de Psicología de la Universidad Veracruzana de México. Antes de presentar la estructura del Programa y los resultados de su aplicación es preciso resumir el estado de la cuestión sobre las teorías del desarrollo humano y sobre la metacognición para nuestro estudio.

\section{Síntesis de las aportaciones de las teorías del desarrollo humano}

De una parte, respecto a las teorías del desarrollo humano se requiere hacer una breve referencia a las teorías psicosociales de la personalidad, a la cognición social y al desarrollo del sí mismo y de la autoestima. Cuando se pretende abordar el desarrollo humano es imprescindible referirse a la personalidad ya que ésta se considera la compendiadora de todos 
los aspectos humanos: el intelecto, las competencias y habilidades, los valores y las actitudes adquiridas en el trascurso de la vida del sujeto, entendiéndose la personalidad como un esquema unificado de las experiencias vividas. Las aportaciones que para nuestro estudio ofrecen las teorías psicosociales, la cognitivo-social, la de las atribuciones y las del desarrollo del sí-mismo o desarrollo humano, son el lugar central que ocupa la autoestima.

Primero, en cuanto a las teorías psicosocilaes de la personalidad, Bandura y Sullivan representan el enfoque teórico del aprendizaje cognitivo-social. Toman de las teorías del aprendizaje la pretensión de estudiar la conducta en su condición moldeable mediante estímulos externos -tales como el medio ambiente o situaciones específicas- y de las teorías cognitivas asumen la idea de que la conducta no depende linealmente de estímulos externos, sino de la elaboración interna de los mismos mediante procesos tales como la valoración, la evocación de la experiencia anterior y la expectativa de futuros acontecimientos y experiencias.

Por una parte, Bandura $(1969,1973,1977,1984,1989)$ se refiere al aprendizaje por observación como aprendizaje «sin ensayo», debido a que el observador aprende sin emitir ninguna actividad abierta. Durante toda la vida tenemos modelos que copiar. El éxito o el fracaso en muchos aspectos de la vida en una cultura depende del aprendizaje por observación. Por otra, Sullivan (1953) concibe a la personalidad como un sistema de energía cuya tarea esencial es la realización de actividades que reduzcan la tensión que le provoca la cultura y las relaciones interpersonales. Su enfoque es esencialmente un enfoque psicosocial del crecimiento de la personalidad que reconoce la singular contribución de las relaciones humanas cambiantes. Si bien no descarta los factores biológicos como condicionantes del desarrollo, los subordina a los determinantes sociales del desarrollo psicológico. Considera además que, ocasionalmente, tales influencias sociales contrarían las necesidades biológicas del individuo, con consecuencias perjudiciales para su personalidad. Finalmente, Mischel (1983, 1986) propone diversas variables de la persona que tienen un papel perdurable en el procesamiento de estímulos en la dirección de la conducta, tales como las habilidades cognoscitivas, expectativas, estrategias y planes de autorregulación. Se puede decir que, los teóricos del aprendizaje cognoscitivo y social, utilizan ampliamente las variables intervinientes de la personalidad, particularmente los determinantes cognoscitivos de la conducta manifiesta.

Segundo, la cognición social hace referencia a las formas de asimilación de las experiencias, relacionando cada una de éstas con los demás y dotándoles de significado. De esta manera, los procesos cognitivos ordenan las ideas propias para formar conceptos y filtrar selectivamente la información según nuestros propósitos y expectativas. En base al cumplimiento de estas expectativas, se verifica su influencia sobre nosotros. Ya Bruner, en 1947 y 1951, destaca dos características básicas de la cognición social: una, el hecho de que la percepción está organizada selectivamente de modo que las nuevas experiencias se asimilan con la previas; y la otra, que los procesos cognitivos operan de modo que, las nuevas experiencias, son asimiladas con mayor facilidad cuando sus componentes son experiencias anteriores que cuando no lo son.

Uno de los problemas cognitivos respecto a nuestra conducta y a la de los demás, es que muy frecuentemente anteponemos nuestras creencias, valores y actitudes; y de esta suerte, erróneamente, consideramos los factores que en realidad tienen gran efecto como inocuos y los que tienen poco efecto los estimamos como influyentes. De hecho, los procesos mentales que controlan nuestra conducta social son distintos a los procesos mentales a través de 
los cuales las explicamos, ya que las ideas preconcebidas guían la manera en que percibimos, interpretamos y recordamos la información. Así, nuestras creencias no sólo controlan nuestras preconcepciones e interpretaciones, sino también nuestros recuerdos; afectando a la reconstruccción de nuestras experiencias, conductas y actitudes pasadas.

Tercero, la teoría de la atribución (Langer, 1989; Wong y Weiner, 1981) ha dado lugar a diferentes explicaciones acerca del por qué de las atribuciones causales, tales como, la oportunidad de demostrar nuestra capacidad para predecir o controlar acontecimientos. Se estima que las atribuciones son bastante estables y resistentes al cambio, existiendo además una tendencia a sobrestimar los mecanismos causales internos.

Cuarto, los factores relacionados con el desarrollo del sí-mismo o desarrollo humano parten de un marco que comprende los conceptos medulares de los humanistas representativos en este campo. El análisis contempla las características que pueden obstaculizar el desarrollo humano, así como aquéllas que conducen a él. Este enfoque resalta los atributos distintivamente humanos, enfatizando el atributo de la libertad. Con respecto a este último punto, debemos matizar la diferencia significativa entre estar libre de controles, reglamentos o restriccciones externas -condiciones que enajenan o mantienen al ser humano en una situación de vida incongruente- y «ser libre» para usar nuestras capacidades, habilidades y recursos en nuestro desarrollo humano, esto es, conseguir la congruencia entre el yo real y el yo ideal para alcanzar el estado de plena realización o autorrealización como ser humano.

En este contexto la autorrealización se entiende únicamente como un estado que proporciona gratificación, como resultado de las capacidades desarrolladas, tales como las de dar amor, el conocimiento o la valoración de algo, capacidades que, a su vez, dependen del desarrollo de la autoestima y de las relaciones interpersonales adecuadas. Es pues, la autorrealización, una consecuencia de una serie de circunstancias controlables y educables que incide en la autoestima. La autoestima, en este marco y con este enfoque, es el punto central -junto con las relaciones interpersonales- para el desarrollo humano. Entre los elementos incidentes en la autoestima están: los valores, la autopercepción, los autoesquemas y las medidas cognitivas que emplean las personas para proteger su autoestima o para incrementarla, y que aquí no podemos explicitar.

\section{Síntesis de las aportaciones de las teorías metacognitivas}

De otra, con relación a la metacognición se precisa, primero, hacer una breve referencia a los elementos básicos que la integran y, en segundo lugar, se apunta la incidencia en el desarrollo humano y en la autoestima.

Según Mayor, Suengas y González (1993), un modelo metacognitivo debe incluir la actividad metacognitiva específica y el objeto propio de esa actividad, el cual es la propia cognición. Proponen a la vez un modelo de esta actividad metacognitiva, constituído por dos componentes básicos: la conciencia y el control del proceso, y un tercer elemento más, la autopoiesis, considerada como un circuito de retroalimentación. En cuanto al objeto de incidencia de la actividad metacognitiva, es decir, la cognición, Mayor (1990) señala a la mente o actividad cognitiva como un todo organizado y dinámico, involucra tres dimensiones indispensables: la información acerca de la utilidad y del cómo va su ejecución paso a paso, el entrenamiento en el cual se enseña a cómo utilizar la estrategia y cómo controlar y evaluar el uso de la misma. 
Centrándonos en la lectura cabe señalar algunas de las estrategias más adecuadas para mejorarla (Repetto y cols; 2002). En primer lugar, se recomienda el «modelo del aprendizaje espontáneo», en el cual el aprendiz es participante activo que elabora hipótesis, adquiere destrezas, y en el que aprender a leer es más que enseñar a leer. En segundo lugar, manifiestan que hay que entender la lectura como un proceso adaptativo: ha de adaptarse a la naturaleza y/o la mayor o menor legibilidad del texto, a las condiciones del sujeto lector -necesidades, capacidad, propósitos- y a las condiciones de la situación. En tercer lugar, ha de fomentarse el desarrollo de las adecuadas habilidades metacognitivas -autoevaluación y autorregulación-, tales como el conocimiento de los aspectos relevantes del texto (si es fácil o difícil, qué elementos o irregularidades contiene, etc.) la aplicación oportuna de las diferentes estrategias, el control de lo que el lector hace y de lo que debe hacer.

En base a esto último podemos señalar que un programa sistemático y global de estrategias metacognitivas para mejorar el rendimiento lector tendría que tener también por objeto la actividad lingüística implicada en la lectura. Por ejemplo, en la actualidad, diversos autores se están abocando al estudio de la habilidad, la cual implica la capacidad para ser conscientes y para controlar la producción y la comprensión de textos. Generalmente se ha vinculado el aprendizaje y la instrucción sobre la lectura a las diferentes concepciones sobre los mecanismos de aprendizaje o los procesos cognitivos implicados en la misma, así como a los distintos sistemas de evaluación. Específicamente, con repecto al desarrollo de estrategias metacognitivas para la mejora de la lectura, debe subrayarse que en los últimos años han aparecido numerosos programas de entrenamiento metacognitivo, como por ejemplo: el programa de Paris y cols. (1986. 1989) y el de Repetto y cols. (2003) que establecen una relación de las estrategias metacognitivas para la lecura que conducen a su comprensión.

El programa de Paris y cols. $(1986,1989)$ tiene como objetivo desarrollar destrezas y estrategias en la activación de los conocimientos previos (conocimiento sobre el conocimiento y pensamiento autodirigido), la elección de las estrategias cognitivas más eficaces y el control de las variables que influyen en el proceso (personales, de tarea y de estrategia. El programa consta de los siguientes apartados: a) consciencia de fines, metas y estrategias de la lectura; b) componentes del significado en el texto; c) habilidades constructivas de comprensión; d) estrategias para controlar y mejorar la comprensión.

El Programa OMECOL de Repetto (1990), más tarde publicado con el título de: «Comprender y Aprender en el Aula» (2003), contiene una serie de estrategias de autoevaluación y autorregulación de la comprensión lectora que pueden enseñarse. Algunas de ellas son: clasificar los propósitos de la lectura, identificar las demandas de la tarea para seleccionar las estrategias adecuadas, planificar las estrategias, usar las claves que proporciona el texto, utilizar las claves contextuales, activar los conoicmientos previos y evaluar el nivel de la comprensión lectora. Repetto (1990) hace énfasis sobre el papel autorregulador que desempeña el lector en cuanto que orienta y dirige sus procesos y elige estrategias adecuadas para los propósitos de la lectura.

\section{Implicaciones de las teorías del desarrollo humano y metacognitivas para la elaboración de un programa metacognitivo}

Pensar sobre lo que pensamos y aprender algunas cosas sobre nosotros mismos para que a su vez aprendamos a actuar en consecuencia, es el paso requerido para desarrollarnos 
como individuos más sanos psicológicamente. Todo lo anterior adquiere aún mayor peso cuando somos conscientes de nuestra experiencia yoica y de nuestra autodirección, esto es, cuando realmente nuestro «sí-mismo» es quien elige las metas así como la forma de alcanzarlas.

El desarrollo humano o desarrollo del sí-mismo, incluye desechar todas las defensas inadecuadas creadas para la proteccción del sí-mismo, así como también -y de suma importancia- incorporar los conocimientos y las habilidades que en su conjunto dirigen a la persona hacia la autoestima y la autorrealización, y que requiere del previo desarrollo metacognitivo en estas áreas. Cuando el individuo se encuentra ante una demanda intrapersonal o interpersonal (social) en la que requiere realizar una actividad que involucra a su sí-mismo, éste necesita enfrentarlo de tal forma que no sólo solucione el problema, sino que además lo satisfaga, le de plenitud e incremente su autoestima. Para ello, la persona debe estar capacitada en habilidades de ajuste en términos generales, del tipo de: la evaluación de la situación al comparala con la información que ya tenemos almacenada en nuestra memoria; la formulación de cursos de acción alternos para manejar la situación; la forma de decisión -eligiendo la alternativa que parece más adecuada- y la decisión de actuación; la utilización de la retroalimentación y la evaluación de la secuencia total; y el acomodo de las conclusiones en el banco de memoria. En todos estos pasos el individuo se percibe como un agente activo con intenciones conscientes y la capacidad para autorregularse y autocontrolarse.

En base a lo anteriormente expuesto, si consideramos que el desarrollo del sí-mismo que un individuo logra puede verse como el nivel de habilidad alcanzado, querrá decir que éste, como producto del aprendizaje (en interacción con el conocimiento y el pensamiento), puede tener niveles muy bajos y que, si además, se le enseña a aprender cómo aprender la habilidad del desarrollo de sí-mismo y, por ende, de una autoestima adecuada, podemos esperar personas con mayor autoconfianza y motivación para el logro de las metas académicas, profesionales y sociales.

Por todo ello, y siguiendo de cerca el Programa de Repetto (1990), el Profesor Carvallo elabora el «Programa de Estrategias Metacognitivas para el Desarrollo Humano» (Carvallo, 1997), del que se ofrece una síntesis a continuación.

\section{Programa de estrategias metacognitivas para el desarrollo humano}

La elaboración del programa parte del análisis de necesidades de los alumnos universitarios, en concreto de los alumnos del primer curso de la Facultad de Psicología de la Universidad Veracruzana (Carvallo, 1999). El programa se destina a entrenar procesos metacognitivos con el objetivo de apreciar si los educandos adquieren un mayor desarrollo humano a través del aprendizaje de las estrategias metacomprensivas. Se diseña con un contenido que pretende la reflexión, el análisis y la discusión sobre casos y situaciones de la maturaleza humana y de interacción social con miras a la construccción de su realidad autoafectiva. Se persigue que el alumno logre el aprendizaje del «conocerse y del pensarse de manera positiva» para desarrollar un sentimiento de autoestima, de tal forma que la falta de asertividad y de autoreconocimiento, cambien, y alcance la conciencia de este cambio y la capacidad para actuar por iniciativa propia, con estrategias de pensamiento positivo sobre sus sentimientos y su valoración personal. Dado el material textual utilizado en el programa 
se hace evidente la necesidad de una lectura comprensiva con la aplicación de las estrategias metacomprensivas seleccionadas.

Los objetivos generales a alcanzar por los alumnos son los siguientes:

- Capacitarles en las estrategias metacomprensivas seleccionadas y así incrementar su comprensión lectora y el «desarrollo del sí-mismo».

- Incrementar la autoestima, autoaceptación, autenticidad y el control respecto a su modo de ser.

- Habituarles en la utilización de estrategias de desarrollo del sí-mismo que deberá emplear de acuerdo a su situación personal y/o social.

- Determinar un nivel consciente y regulador de los procesos inmersos en la destreza de desarrollo del sí-mismo y más concretamente en el fenómeno de la comprensión, desarrollo de la autoestima y de la seguridad personal.

- Mejorar, mediante la aplicación del programa, el desarrollo del sí-mismo.

El programa se estructura en cinco Módulos, divididos a su vez en Unidades. En el siguiente cuadro se resume el nombre de cada uno de los Módulos así como el de las Unidades de las que constan.

EL programa consta de la guía del profesor y del cuaderno del alumno. La guía del profesor, explicita los objetivos generales a alcanzar en cada uno de los Módulos propuestos, así como la descripción de las técnicas de orientación metacognitiva. Del mismo modo, se detalla en cada unidad el objetivo a trabajar, la temporalización y la formación de grupos de

\section{Cuadro resumen de la estructura del Programa}

\begin{tabular}{|c|c|}
\hline $\begin{array}{c}\text { MÓDULO I: } \\
\text { Búsqueda de información adecuada y } \\
\text { construccción del significado: el lector como } \\
\text { un investigador privado }\end{array}$ & $\begin{array}{l}\text { Unidad 1: Busca información adecuada y construye } \\
\text { el significado } \\
\text { Unidad 2: Encuentra el significado: el lector como un } \\
\text { investigador privado }\end{array}$ \\
\hline $\begin{array}{c}\text { MÓDULO II: } \\
\text { Las impresiones del significado del texto y } \\
\text { su aplicación al desarrollo personal }\end{array}$ & $\begin{array}{l}\text { Unidad 1: Implicación del signifcado en la vida cotidia } \\
\text { Unidad 2: Despertar las impresiones }\end{array}$ \\
\hline $\begin{array}{l}\text { MÓDULO III: } \\
\text { Soy competente para vivir y digno de ser } \\
\text { feliz: el camnio hacia la autoestima }\end{array}$ & $\begin{array}{l}\text { Unidad 1: Entendiendo la autoestima } \\
\text { Unidad 2: Conociendo mi sí-mismo niño que llevo dentro } \\
\text { Unidad 3: Integrando mi pasado y mi presente } \\
\text { Unidad 4: Saber qué hacer y tener conciencia sobre ello }\end{array}$ \\
\hline $\begin{array}{l}\text { MÓDULO IV: } \\
\text { Confiando en la eficacia de mi mente: } \\
\text { eliminando más obstáculos y pensando } \\
\text { conscientemente me dirijo hacia mi plenitud }\end{array}$ & 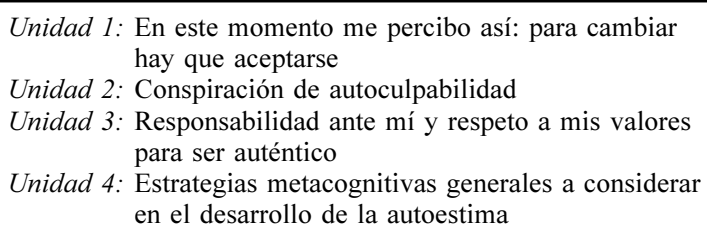 \\
\hline $\begin{array}{l}\text { MÓDULO V: } \\
\text { Relaciones interpersonales efectivas: } \\
\text { cómo comunicarse sin hacerse daño }\end{array}$ & $\begin{aligned} & \text { Unidad 1: } \text { Las cosas mal dichas afectan a nuestras } \\
& \text { relaciones personales } \\
& \text { Unidad 2: Aprendiendo a ser positivo en mi comunicación } \\
& \text { Unidad 3: Continuando con mi comunicación positiva: } \\
& \\
& \text { cómo recibir las críticas }\end{aligned}$ \\
\hline
\end{tabular}


trabajo, los materiales a emplear y el desarrollo de la orientación metacognitiva. El cuaderno del alumno recoge las actividades de cada unidad agrupadas por lecturas y los ejercicios correspondientes.

\section{Evaluación del Programa}

Tomando en consideración la importancia de la evaluación de los programas de orientación y el hecho de que cualquier programa es siempre susceptible de mejoras, el proceso de investigación seguido pretende primero, valorar la calidad de su contenido y, segundo, los logros del programa tras su aplicación definitiva. Así se persigue un doble fin: por un lado, reducir el riesgo de fracaso en su puesta en marcha aplicándolo en las condiciones más favorables y, por otro, la evaluación de los resultados que proporciona. Antes de la elaboración del programa se hizo el análisis de necesidades del contexto y de los alumnos de la Facultad de Psicología de la Universidad Veracruzana. Después de la elaboración del programa se somete a su validación. Para ello, la fase de evaluación incial del programa parte de una validación de expertos -tanto de un carácter teórico y práctico- en base a las dimensiones de la «propuesta evaluativa de programas de orientación» del profesor Pérez Juste (1991). Tras la fase de decisiones de mejora se realizan los cambios pertinentes hasta completar finalmente el programa para aplicarlo a los alumnos. Tras la revisión del programa y a la luz de los resultados se afirma que el programa cuenta con la calidad técnica y la coherencia para su viabilidad, a la vez que da respuesta a las necesidades y carencias de sus destinatarios.

El problema en esta fase de evaluación inicial cabe formularlo como sigue:

\section{Formulación del problema}

Los interrogantes de los que se parte en el estudio son:

- El contenido del programa de desarrollo del sí mismo a través de estrategias metacognitivas, ¿es cohrente con las bases que lo sustentan, adecuado para los destinatarios y relevante para ser evaluado posteriormente?

- ¿Existe congruencia entre el programa y las necesidades y carencias de los destinatarios?

- Las características del programa y las condiciones con que cuenta son adecuadas para ponerlo en marcha?

Tras concretar las variables de estudio seleccionadas y la muestra, se define el diseño de investigación y los instrumentos de medida para la evaluación del mismo en sus resultados.

\section{Formulación de hipótesis}

Sobre la base del problema planteado, la hipótesis de investigación de la evaluación del programa en sus resultados se formula del modo siguiente: El desarrollo personal de los alumnos del primer semestre de la Licenciatura en Psicología se incrementará como resulta- 
do de la implementación del Programa de Estrategias Metacognitivas para el Desarrollo Humano (Carvallo, 1997)

Las hipótesis estadísticas, nula y alterna, son las siguientes.

Hipótesis de nulidad-Ho-: No existe diferencia significativa entre los promedios de las puntuaciones obtenidas en el inventario (o escala), por los estudiantes a quienes se les aplica el Programa de Estrategias Metacognitivas para el Desarrollo Humano a través respecto a aquellos estudiantes a quienes no se les aplica dicho programa.

Hipótesis alterna-Hi-: Los promedios de las puntuaciones obtenidas en el inventario (o escala), son significativamente favorables en aquellos alumnos en que se aplica el Programa de Estrategias Metacognitivas para el Desarrollo Humano.

Respecto a las variables seleccionadas, la Variable Independiente (VI) es la intervención o no intervención del Programa de Estrategias Metacognitivas para el Desarrollo Humano en los alumnos del primer semestre de la Licenciatura en Psicología de la Universidad Veracruzana. La Variable Dependiente (VD) valora los efectos de la VI es el desarrollo personal alcanzado, el cual, es medido a través de las puntuaciones obtenidas por los alumnos en los rasgos del CPI (especialmente los asociados a la autoestima) al término de la fase experimental.

\section{Población y muestra}

En la presente investigación, la población con la cual se trabaja es la de los estudiantes recién ingresados a la Facultad de Psicología de la Universidad Veracruzana. Dado que la matrícula anual de ingreso al primer semestre en esta Facultad es de 120 alumnos, estos se distribuyen en los cuatro grupos existentes asignandolos de manera aleatoria.

\section{Diseño de la investigación}

Para el control de las variables extrañas se empleó el diseño de grupo de control pretestpostest con grupos aleatorios que se muestra en la Tabla 1.

TABLA 1: Diseño de la investigación.

\begin{tabular}{|c|c|c|c|c|}
\hline & Grupo & Pretest & $\begin{array}{c}\text { Variable } \\
\text { independiente }\end{array}$ & Postest \\
\hline$(\mathrm{R})$ & 1 & $\mathrm{y}_{1}$ & $\mathrm{VI}$ & $\mathrm{y}_{2}$ \\
\hline$(\mathrm{R}))$ & 2 & $\mathrm{y}_{3}$ & & $\mathrm{y}_{4}$ \\
\hline
\end{tabular}

Para la evaluación del desarrollo humano en los alumnos se aplica el inventario de la Configuración Psicológica Individual-CPI- (Gough, 1990). Éste permite medir los rasgos relacionados con los factores involucrados en el desarrollo afectivo-emocional, dado que se centra en las características de personalidad que tienen una amplia aplicabilidad al comportamiento humano y que están relacionadas con los aspectos favorables, deseables y positivos de la personalidad, tal y como lo menciona su propio autor. 
A continuación se exponen los rasgos que contempla el CPI:
1. Do (Dominancia).
2. Cc (Capacidad de Categoría).
3. Sd (Sociabilidad).
4. Ps (Presencia Social).
5. Aa (Autoaceptación).
6. Sb (Sentido de Bienestar).
7. Re (Responsabilidad).
8. Sn (Socialización).
9. Ac (Autocontrol)
10. To (Tolerancia).
11. Bi (Buena Impresión).
12. Co (Comunalidad).
13. Lc (Logro Conformidad).
14. Li (Logro Independencia).
15. Ei (Eficacia Intelectual).
16. Sp (Sentido Psicológico).
17. Fx (Flexibilidad).
18. Fe (Feminidad).

El procedimiento de la investigación consiste en la aplicación del «Programa de Estrategias Metacognitivas para el Desarrollo Humano» en el grupo experimental y la no aplicación en el grupo control. Esto se lleva a cabo, como se ha indicado, en las aulas de la Facultad de Psicología de la Universidad Veracruzana (México), durante cuatro meses, en dos sesiones por semana, de dos horas y media cada una, dirigidas y supervisadas por el investigador-profesor responsable de los dos grupos, el mismo que se encargó de llevar a cabo las evaluaciones de pre y post intervención en ambos grupos. A conitnuación se ofrece una síntesis de los análisis y la discusión de los resultados correspondientes.

\section{Análisis y discusión de los resultados}

Primero se procede a la calificación del inventario CPI. La puntuación se obteine de forma directa y posteriormente se convierte en puntuación estándar.

Para la inferencia estadística se realizó un análisis por medio de las pruebas multivariadas Lambda de Wilk's y Traza de Pillai-Bartlett. Se presenta primero el análisis de los datos del diseño y después el análisis entre las relaciones secundarias.

\section{Análisis de los datos del diseño}

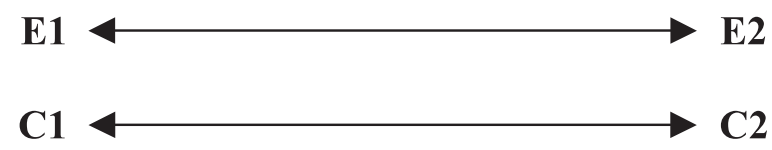

Basado en las medias de los dieciocho rasgos estudiados se presenta el perfil de medias de los dos grupos, control y experimental en la figura 1. En ella pueden visualizarse los cambios existentes entre los grupos en los diferentes rasgos.

En la gráfica podemos observar uno a uno los cambios o no cambios aparentes entre los grupos.

En una etapa posterior se procede a un análisis centrado en los cambios entre la medición antes-después de cada uno de los rasgos en los dos grupos experimental y control. Con objeto de una mejor visualización de los posibles cambios se presenta una gráfica de cajas y patas de los rasgos en la condición antes-después, tanto para el grupo experimental como para el grupo control. 


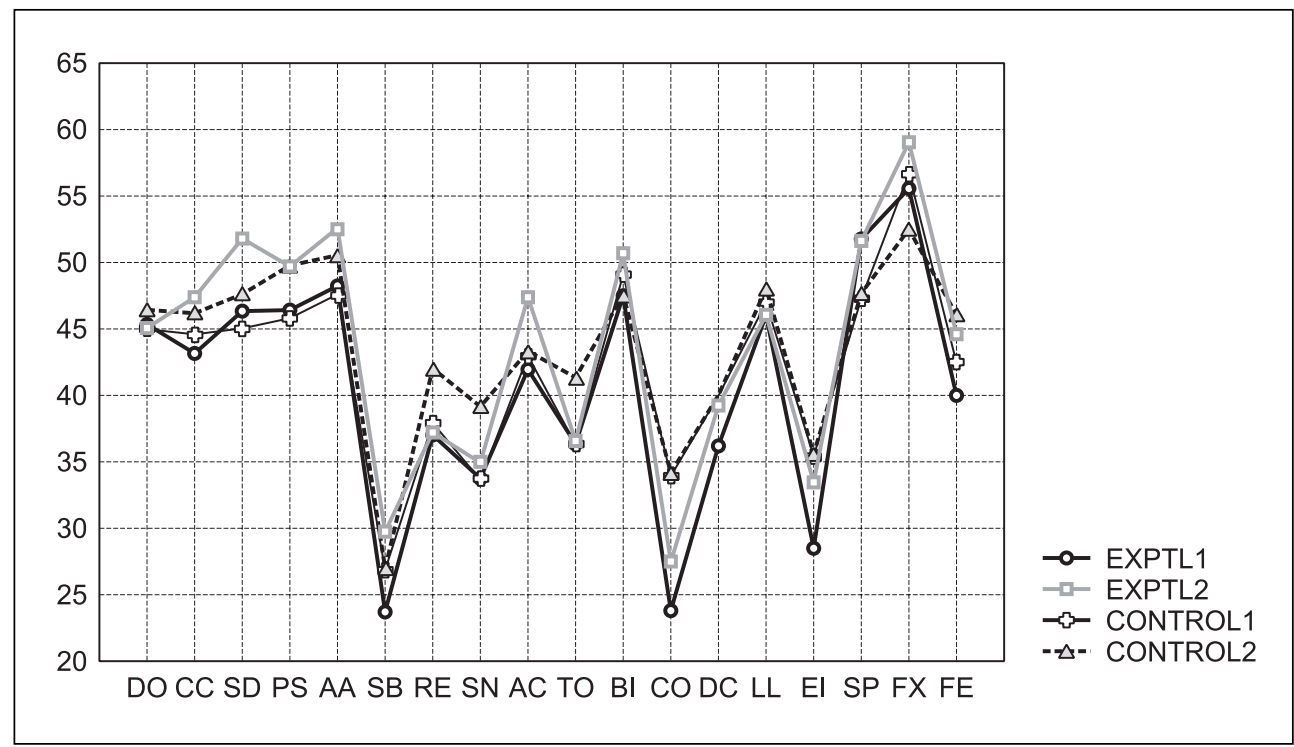

FIGURA 1.
Perfil de medias en los pretest y postest de los dos grupos.

Para la inferencia estadística se realiza un análisis multivariado, por medio de las pruebas estadísticas Lambda de Wilk's y Traza de Pillai-Bartlett.

En este análisis multivariado, las diferencias entre el total de rasgos para el grupo experimental (antes-después) resultan ser significativas, $\mathrm{p}<0.005$, según se oberva en la Tabla 2.

Por otra parte el análisis multivariado con las mismas pruebas estadísticas en el grupo control (antes-después) entre los diversos rasgos apenas muestra diferencias significativas, con una $\mathrm{p}<0.10$ (Tabla 3 ).

A continuación se realiza un análisis especifico de los cambios ocurridos o no ocurridos en cada uno de los rasgos.

Con respecto al rasgo Dominancia (DO) se observa como la media de aplicaiones se aglutinan alrededor de las medias 45 y 46 (fig. 1), refiriendo esto una nula diferencia entre las cuatro medias. Así mismo observamos la ausencia de diferencia entre las cajas tanto en el grupo experimental (fig. 2) como en el grupo control (fig. 3).

En cuanto al rasgo Capacidad de Categoría (CC) observamos en el perfil de medias un ligero cambio en el grupo experimental entre la medición antes-después (fig. 1); el mismo efecto puede verse en las cajas de dispersión del grupo experimental (fig. 2). Sin embargo estadísticamente no resulta significativo este cambio.

En el rasgo Sociabilidad (SD) sí encontramos una clara diferencia entre la media obtenida en el pretest y la media obtenida en el postest, aspecto que no se presenta en el grupo control. En las cajas también es clara la separación de la distribución de la variabilidad para el grupo experimental en la medición antes-después (fig. 2), situación que no ocurre en el grupo control (fig. 3). El cambio observado en el grupo experimental para este rasgo tuvo 


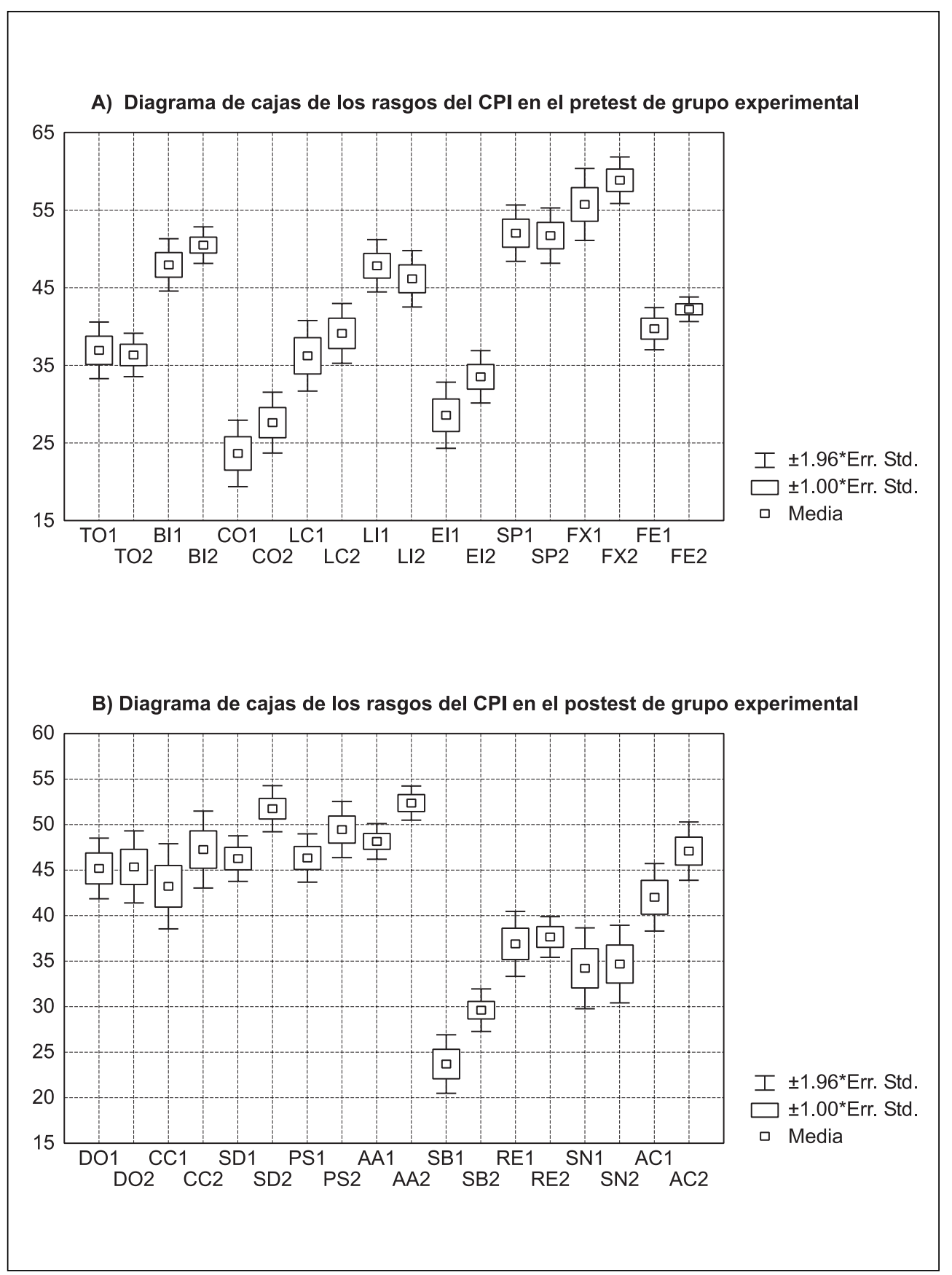

FIGURA 2. 


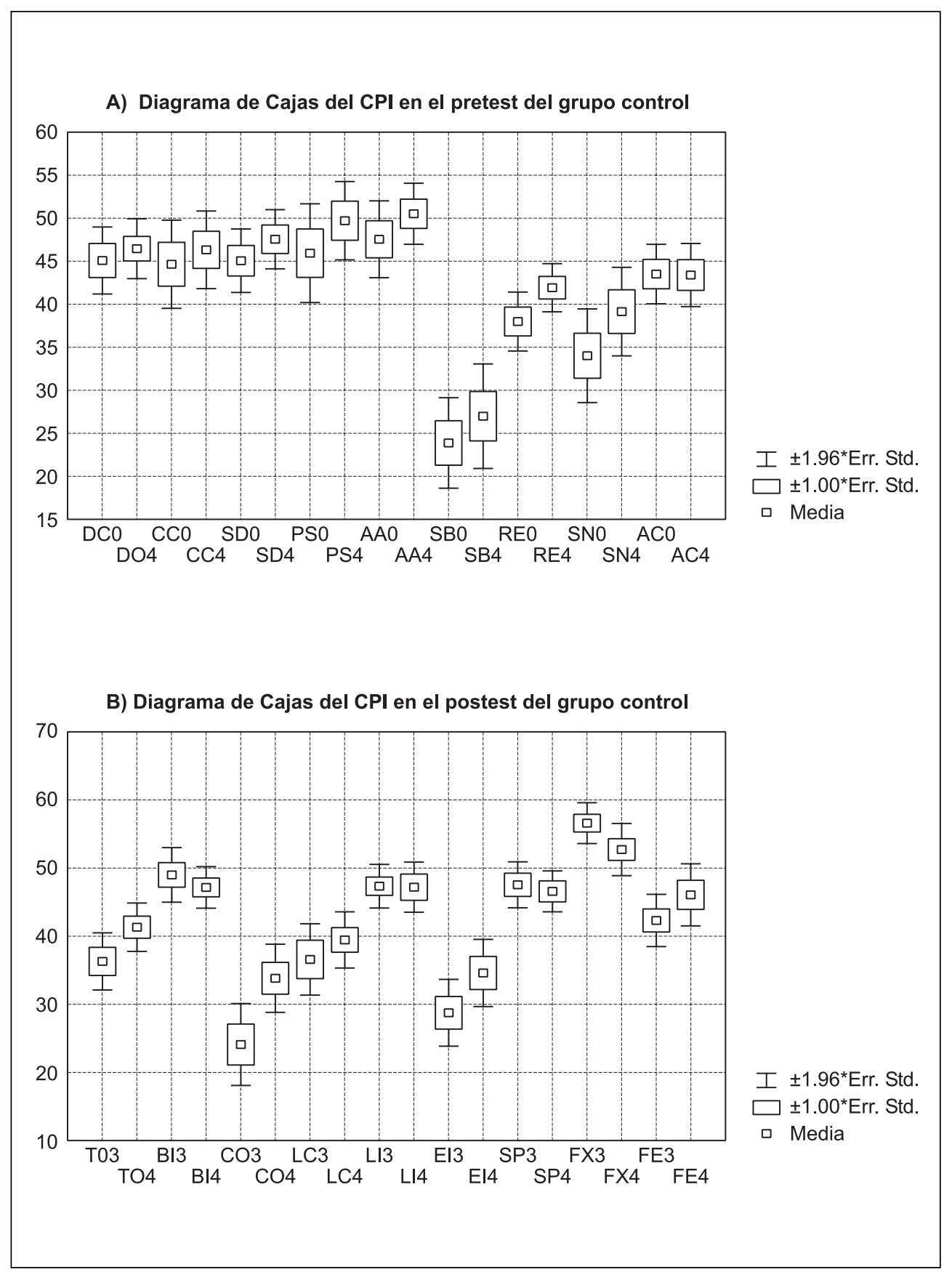

FIGURA 3. 
TABLA 2: Prueba de significación (anova manova) entre los E1-E2.

\begin{tabular}{|l|c|c|c|c|}
\hline & $\begin{array}{c}\text { Efecto de la media } \\
\text { cuadrada }\end{array}$ & $\begin{array}{c}\text { Error de la media } \\
\text { cuadrada }\end{array}$ & F 1,52 & Nivel-p \\
\hline DO & 0.0000 & 91.2179 & 0.00000 & 1.000000 \\
\hline CC & 220.0185 & 138.6937 & 1.58636 & 0.213471 \\
\hline SD & 411.1296 & 45.9174 & 8.95368 & 0.004228 \\
\hline PS & 140.1667 & 58.9487 & 2.37777 & 0.109137 \\
\hline AA & 253.5 & 23.9858 & 10.56877 & 0.002021 \\
\hline SB & 480.0185 & 53.2365 & 9.01672 & 0.004106 \\
\hline RE & 7.4074 & 64.151 & 0.11547 & 0.735371 \\
\hline SN & 4.1667 & 136.0128 & 0.03063 & 0.861738 \\
\hline AC & 362.963 & 85.2407 & 4.25809 & 0.044072 \\
\hline TO & 6.6852 & 70.7835 & 0.09445 & 0.759827 \\
\hline BI & 88.1667 & 59.1909 & 1.48953 & 0.227795 \\
\hline CO & 200.2963 & 119.7835 & 1.67215 & 0.201686 \\
\hline LC & 121.5 & 124.5712 & 0.97535 & 0.327924 \\
\hline LI & 39.1852 & 83.3262 & 0.47026 & 0.495913 \\
\hline EI & 337.5 & 110.2507 & 3.0612 & 0.086082 \\
\hline SP & 1.5 & 89.2806 & 0.0168 & 0.897368 \\
\hline FX & 140.1667 & 106.114 & 1.32091 & 0.255688 \\
\hline FE & 80.6667 & 32.3661 & 2.49232 & 0.120468 \\
\hline & & & & \\
\hline
\end{tabular}

\begin{tabular}{|l|c|c|}
\hline \multicolumn{1}{|c|}{ Prueba } & Valor & Nivel-p \\
\hline Lambda de Wilks' Rao R Form 2 (18,35) & $\begin{array}{l}0.397183 \\
2.951147\end{array}$ & 0.002983 \\
\hline Traza de Pillai-Bartlett V (18,35) & $\begin{array}{l}0.602817 \\
2.951147\end{array}$ & 0.002983 \\
\hline
\end{tabular}

una $\mathrm{p}<0.01$ (tabla 1 ); en el grupo control no hubo ningún cambio significativo ya que obtuvo una $\mathrm{p}>0.10$.

La Presencia Social (PS) resulta con un cambio visible en las medias de las mediciones antes-después del grupo experimental (fig. 1), pero no lo suficiente para ser significativo ya que aunque tiene una probabilidad extremadamente cercana a 0.10 , esta resulta ser ligeramente mayor (0.1091) (tabla 1). El grupo control presenta también cambios insignificantes con una $\mathrm{p}>0.10$ (tabla 2). El diagrama de cajas permiten ver también como las dispersiones tienden a separarse mas en el grupo experimental (fig. 3) que en el grupo control (fig. 4).

Un rasgo importante en esta investigación es el de Auto-aceptación (AA), el cual resulta con cambios significativos en el grupo experimental con una $\mathrm{p}=0.002$ (tabla 1), mas no en el grupo control, ya que sus cambios tienen una $p>0.10$ (tabla 2). La diferencia de ambos grupos puede verse en el perfil de medias en donde se observan claramente los cambios entre los puntuaciones medias de E1 y E2. De igual forma en las cajas y patas del grupo ex- 
TABLA 3: Prueba de significación (anova, manova) entre los grupos C1-C2.

\begin{tabular}{|l|c|c|c|c|}
\hline & $\begin{array}{c}\text { Efecto de la media } \\
\text { cuadrada }\end{array}$ & $\begin{array}{c}\text { Error de la media } \\
\text { cuadrada }\end{array}$ & F 1,52 & Nivel-p \\
\hline DO & 21.780 & 92.6000 & 0.235205 & 0.629897 \\
\hline CC & 32.000 & 153.9583 & 0.207848 & 0.650515 \\
\hline SD & 79.380 & 79.2967 & 1.001051 & 0.322074 \\
\hline PS & 188.180 & 173.7300 & 1.083175 & 0.303202 \\
\hline AA & 106.580 & 104.9667 & 1.015370 & 0.318673 \\
\hline SB & 106.580 & 204.8050 & 0.520397 & 0.474172 \\
\hline RE & 184.320 & 64.2367 & 2.869389 & 0.096760 \\
\hline SN & 343.220 & 181.9367 & 1.886481 & 0.175981 \\
\hline AC & 0.320 & 81.8683 & 0.003909 & 0.950409 \\
\hline TO & 297.680 & 95.2300 & 3.125906 & 0.083413 \\
\hline BI & 48.020 & 77.5583 & 0.619147 & 0.435232 \\
\hline CO & 1200.50 & 199.9350 & 6.004451 & 0.017966 \\
\hline LC & 98.000 & 146.4567 & 0.669140 & 0.417394 \\
\hline LI & 0.320 & 78.1833 & 0.004093 & 0.949255 \\
\hline EI & 386.420 & 156.0450 & 2.476337 & 0.122140 \\
\hline SP & 8.820 & 68.4617 & 0.128831 & 0.721221 \\
\hline FX & 188.180 & 78.7983 & 2.388122 & 0.128829 \\
\hline FE & 169.280 & 116.1133 & 1.457886 & 0.233183 \\
\hline
\end{tabular}

\begin{tabular}{|l|c|c|}
\hline \multicolumn{1}{|c|}{ Prueba } & Valor & Nivel-p \\
\hline Lambda de Wilks' Rao R Form 2 (18,31) & $\begin{array}{l}0.643179 \\
0.955451\end{array}$ & 0.527942 \\
\hline Traza de Pillai-BartlettV (18,31) & $\begin{array}{l}0.356821 \\
0.955451\end{array}$ & 0.527942 \\
\hline
\end{tabular}

perimental se aprecia la marcada separación de ambas distribuciones, cosa que no ocurre en el grupo control. (fig. 2 y fig. 3).

Otro rasgo fundamental en la investigación es el Sentido de Bienestar (SB) el cual obtiene cambios significativos en el grupo experimental con una $\mathrm{p}=0.004$ (tabla 1). El grupo control no observó cambios significativos al obtener una $\mathrm{p}>0.10$ (tabla 2). Los cambios manifestados pueden verse en el perfil de medias en donde se observa cómo el cambio en el grupo experimental supera al cambio presentado en el grupo control (fig. 1). De igual manera las cajas en el grupo experimental dan cuenta de la separación significativa entre ambas distribuciones de variabilidad (fig. 2), en tanto que en el grupo control de hecho estas distribuciones se sobreponen una a otra (fig. 3), denotando un ligero cambio no significativo.

Apenas muestra diferencias y menos aún significativas las variables de: Responsabilidad y Socialización (SN). Referente al rasgo denominado Autocontrol (AC) puede observarse en el perfil de medias (fig. 1) cómo, en tanto que el grupo control de hecho no cam- 
bia, en el grupo experimental se aprecia un cambio sustancial. En relación a las cajas correspondientes a este rasgo se observa también en el grupo experimental un desplazamiento de la condición postest respecto de la condición pretest cercana al límite de la primera desviación (fig. 2), en tanto que en el grupo control las condiciones pretest-postest permanecen casi superpuestas (fig. 3).El nivel de significación obtenido en el cambio del grupo experimental fue de una $\mathrm{p}<0.05$ (tabla 1) en tanto que para el grupo control se obtuvo una $\mathrm{p}>0.10$ (tabla 2).

El rasgo Tolerancia (TO) no presenta cambios visibles entre las medias del grupo experimental en las condiciones pretest-postest. Así mismo, el rasgo Buena Impresión (BI), muestra en el perfil de medias puntuaciones muy cercanos entre sí, tanto para el grupo control como para el experimental (fig. 1). El rasgo Comunalidad (CO) tampoco presenta cambios significativos, mientras que los rasgos Logro Conformidad (LC), Logro Indepedendencia (LI) y Eficacia Intelectual (EI) alcanzan ligeros cambios en los grupos, experimental y control, pero sin ser significtaivos.

Con relación al rasgo Sentido Psicológico (SP), observamos en las cajas, tanto para el grupo experimental como para el control, un casi total solapamiento de las distribuciones (figs. 2 y 3), sobre todo en el grupo experimental. El perfil de medias (fig. 1), por su parte, da cuenta de la forma en que las medias para ambos grupos en las dos condiciones antesdespués se mantienen casi constantes. Los relativos cambios observados entre las dos aplicaciones, pretest postest, para ambos grupos resultan no significativos con una $\mathrm{p}>0.10$ (tablas 1 y 2 ).

Por último, con respecto al rasgo Flexibilidad (FX) encontramos cambios no significativos en ambos grupos con una $p>0.10$ (tablas 1 y 2) y el perfil de medias (fig. 1) nos brinda información del ligero cambio que se observa en el grupo experimental; en tanto que el rasgo Feminidad (FE) presenta unos ligeros cambios en las medias de las aplicaciones pretest-postest en ambos grupos (fig. 2) pero no son significativos para ambos grupos al poseer una $\mathrm{p}>0.10$ (tablas $1 \mathrm{y} 2$ ).

\section{Análisis entre las relaciones secundarias del diseño}

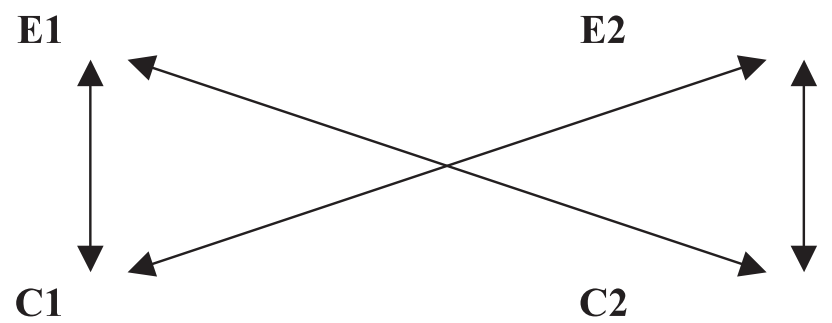

Este análisis comparativo hace referencia a las diferentes condiciones de medición entre el grupo control y el grupo experimental.

La comparación entre la primera aplicación (pretest) del grupo experimental (E1) y del grupo control (C1) con las pruebas Lambda y Bartlett dan como resultado en sus efectos principales diferencias nulas con una $\mathrm{p}>0.10$ (tabla 4). En el análisis por rasgo encontra- 
mos que no existen diferencias significativas en ninguno de ellos con una $p>0.10$ a excepción del rasgo Sentido Psicológico, el cual resulta también no significativo con una $p>0.05$ (tabla 4).

TABLA 4: Prueba de significación (anova, manova), entre los grupos E1-C1.

\begin{tabular}{|l|c|c|c|c|}
\hline & $\begin{array}{c}\text { Efecto de la media } \\
\text { cuadrada }\end{array}$ & $\begin{array}{c}\text { Error de la media } \\
\text { cuadrada }\end{array}$ & F (df1,2) 1,50 & Nivel-p \\
\hline DO & 0.1356 & 88.8261 & 0.001527 & 0.968984 \\
\hline CC & 28.7756 & 161.6133 & 0.178052 & 0.674861 \\
\hline SD & 18.2336 & 62.2815 & 0.292761 & 0.590859 \\
\hline PS & 3.2741 & 128.0741 & 0.025564 & 0.873614 \\
\hline AA & 3.4701 & 73.0641 & 0.047494 & 0.828369 \\
\hline SB & 0.5908 & 122.6928 & 0.004815 & 0.944956 \\
\hline RE & 14.9751 & 84.1170 & 0.178027 & 0.674883 \\
\hline SN & 1.2564 & 167.8933 & 0.007483 & 0.931409 \\
\hline AC & 31.3505 & 87.3618 & 0.358858 & 0.551846 \\
\hline TO & 3.1026 & 99.1733 & 0.031284 & 0.860322 \\
\hline BI & 18.3249 & 87.0073 & 0.210613 & 0.648276 \\
\hline CO & 2.4962 & 182.4516 & 0.013681 & 0.907355 \\
\hline LC & 4.1574 & 162.9122 & 0.025519 & 0.873724 \\
\hline LI & 1.5600 & 71.7888 & 0.021730 & 0.883399 \\
\hline EI & 4.5584 & 144.3304 & 0.031583 & 0.859664 \\
\hline SP & 279.1140 & 82.4593 & 3.384871 & 0.071737 \\
\hline FX & 16.3262 & 103.4900 & 0.157756 & 0.692921 \\
\hline FE & 88.8534 & 70.7414 & 1.256032 & 0.267758 \\
\hline & & & & \\
\hline
\end{tabular}

\begin{tabular}{|l|c|c|}
\hline \multicolumn{1}{|c|}{ Prueba } & Valor & Nivel-p \\
\hline Lambda de Wilks' Rao R Form 2 (18,33) & $\begin{array}{l}0.772628 \\
0.539521\end{array}$ & 0.91633 \\
\hline Traza de Pillai-Bartlett V (18,33) & $\begin{array}{l}0.227372 \\
0.539521\end{array}$ & 0.91633 \\
\hline
\end{tabular}

El análisis multivariado entre las puntuaciones de la primera aplicación del grupo experimental (E1) y los de la segunda aplicación del grupo control (C2) da como resultado en sus efectos globales, como era de esperar, diferencias no significativas con una $p>0.10$. Sin embargo en algunos rasgos individuales tales como Responsabilidad (RE) Comunalidad (CO), Sentido Psicológico (SP) y Feminidad (FE) se observan cambios significativos con una $\mathrm{p}<0.05$, que deberán atribuirse a características propias del grupo control, en cuanto al aspecto escolar (Tabla 5).

En la comparación entre el postest del grupo experimental (E2) y el pretest del grupo control (C1) aunque encontramos en el análisis multivariado que las diferencias son nulas 
TABLA 5: Prueba de significación (anova manova), entre los grupos E1-C2.

\begin{tabular}{|l|c|c|c|c|}
\hline & $\begin{array}{c}\text { Efecto de la media } \\
\text { cuadrada }\end{array}$ & $\begin{array}{c}\text { Error de la media } \\
\text { cuadrada }\end{array}$ & F 1,50 & Nivel-p \\
\hline DO & 19.250 & 81.1365 & 0.237258 & 0.628323 \\
\hline CC & 123.853 & 142.2933 & 0.870403 & 0.355327 \\
\hline SD & 23.128 & 58.8063 & 0.393295 & 0.533426 \\
\hline PS & 148.103 & 90.6533 & 1.633724 & 0.207091 \\
\hline AA & 74.954 & 53.2625 & 1.407257 & 0.241119 \\
\hline SB & 127.442 & 148.1600 & 0.860167 & 0.358148 \\
\hline RE & 313.461 & 71.9442 & 4.357003 & 0.041974 \\
\hline SN & 315.354 & 157.1125 & 2.007186 & 0.162756 \\
\hline AC & 25.227 & 95.8658 & 0.263153 & 0.610221 \\
\hline TO & 250.293 & 82.9941 & 3.015795 & 0.088615 \\
\hline BI & 7.733 & 70.3849 & 0.109872 & 0.741675 \\
\hline CO & 1360.739 & 148.0756 & 9.189484 & 0.003850 \\
\hline LC & 147.065 & 128.4802 & 1.144651 & 0.289809 \\
\hline LI & 3.332 & 85.2672 & 0.039081 & 0.844090 \\
\hline EI & 491.379 & 146.3736 & 3.357021 & 0.072878 \\
\hline SP & 389.396 & 77.3825 & 5.032100 & 0.029343 \\
\hline FX & 98.776 & 123.0660 & 0.802628 & 0.374602 \\
\hline FE & 514.601 & 91.1926 & 5.643015 & 0.021400 \\
\hline & & & & \\
\hline
\end{tabular}

\begin{tabular}{|c|l|c|}
\hline \multicolumn{1}{|c|}{ Prueba } & \multicolumn{1}{|c|}{ Valor } & Nivel-p \\
\hline Lambda de Wilks' Rao R Form 2 (18,33) & $\begin{array}{l}0.562608 \\
1.4253\end{array}$ & 0.184019 \\
\hline Traza de Pillai-Bartlett V (18,33) & $\begin{array}{l}0.437392 \\
1.4253\end{array}$ & 0.184019 \\
\hline
\end{tabular}

con una $\mathrm{p}>0.10$, a nivel univariado se hallan diferencias significativas con una $\mathrm{p}<0.05$ en tres de los principales rasgos asociados a la autoestima, siendo estos, la Autoaceptación (AA), el Sentido de Bienestar (SB) y la Sociabilidad (SD), este último obtuvo una diferencia significativa con una $\mathrm{p}<0.005$ (tabla 6).

En el análisis multivariado para las diferencias entre los puntuaciones del postest tanto del grupo experimental (E2) como del grupo control (C2) encontramos que esta diferencia es significativa con una $\mathrm{p}<0.01$ (tabla 7 ).

En el análisis univariado observamos diferencias significativas, con una $p<0.05$, en dos rasgos asociados de alguna manera a la autoestima y que son Flexibilidad (FX) y Tolerancia (TO) y otros dos con una $\mathrm{p}<0.10$ que son la Sociabilidad (SD), y la Buena Impresión (BI). Existen otros tres rasgos significativos también con $\mathrm{p}<0.05$ pero probablemente más asociados a los aspectos escolares, estos son Responsabilidad (RE), Sentido Psicológico (SP) y Comunalidad (CO) (Tabla 6). 
TABLA 6: Prueba de significación (anova-manova)entre los grupos E2-C1.

\begin{tabular}{|l|c|c|c|c|}
\hline & $\begin{array}{c}\text { Efecto de la media } \\
\text { cuadrada }\end{array}$ & $\begin{array}{c}\text { Error de la media } \\
\text { cuadrada }\end{array}$ & F 1,50 & Nivel-p \\
\hline DO & 0.1356 & 102.6261 & 0.001322 & 0.971144 \\
\hline CC & 84.2849 & 149.7481 & 0.562844 & 0.456632 \\
\hline SD & 583.3511 & 65.0726 & 8.964622 & 0.004271 \\
\hline PS & 180.0626 & 137.4341 & 1.310174 & 0.257813 \\
\hline AA & 305.3868 & 72.4507 & 4.215096 & 0.045316 \\
\hline SB & 429.1214 & 103.8187 & 4.133372 & 0.047372 \\
\hline RE & 1.4423 & 56.4400 & 0.025555 & 0.873637 \\
\hline SN & 9.7500 & 159.0000 & 0.061321 & 0.805435 \\
\hline AC & 171.1510 & 71.3781 & 2.397808 & 0.127812 \\
\hline TO & 0.5990 & 82.0415 & 0.007301 & 0.932247 \\
\hline BI & 24.2715 & 65.6296 & 0.369825 & 0.545852 \\
\hline CO & 151.2369 & 168.4368 & 0.897885 & 0.347907 \\
\hline LC & 76.9074 & 141.6722 & 0.542854 & 0.464695 \\
\hline LI & 23.9047 & 76.4481 & 0.312692 & 0.578529 \\
\hline EI & 252.1546 & 118.0904 & 2.135268 & 0.150202 \\
\hline SP & 240.4281 & 81.1926 & 2.961207 & 0.091468 \\
\hline FX & 57.2856 & 62.9389 & 0.910178 & 0.344657 \\
\hline FE & 0.3834 & 53.9369 & 0.007108 & 0.933149 \\
\hline & & & & \\
\hline
\end{tabular}

\begin{tabular}{|c|l|c|}
\hline \multicolumn{1}{|c|}{ Prueba } & Valor & Nivel-p \\
\hline Lambda de Wilks' Rao R de 2 (18,33) & $\begin{array}{l}0.55437 \\
1.473723\end{array}$ & 0.162986 \\
\hline Traza de Pillai-Bartlett V $(18,33)$ & $\begin{array}{l}0.44563 \\
1.473723\end{array}$ & 0.162986 \\
\hline
\end{tabular}

\section{Conclusiones}

En la presente investigación observamos con respecto a los cambios entre el pretest y el postest del grupo experimental (E1, E2) que en el análisis multivariable se produjo una diferencia significativa extrema, lo cual en términos generales implica cambios en la personalidad.

En el análisis de los rasgos hallamos que varios de estos presentan cambios significativos relacionados con los dos elementos principales de la autoestima: la eficacia personal y el sentido del mérito personal. Con respecto al primer componente, la autoeficacia, encontramos los rasgos de autocontrol y sociabilidad y, extremadamente cercano, el rasgo de presencia social. Respecto al segundo componente, el sentido de mérito, hallamos cambios en los rasgos de autoaceptaión y en el sentido de bienestar. Lo anterior nos lleva a concluir que se logran cambios en el sentido esperado. El análisis multivariado para los cambios del 
TABLA 7: Prueba de significación (anova manova), entre los grupos E2-C2.

\begin{tabular}{|l|c|c|c|c|}
\hline & $\begin{array}{c}\text { Efecto de la media } \\
\text { cuadrada }\end{array}$ & $\begin{array}{c}\text { Error de la media } \\
\text { cuadrada }\end{array}$ & F 1,50 & Nivel-p \\
\hline DO & 19.2503 & 94.9365 & 0.20277 & 0.654441 \\
\hline CC & 11.6695 & 130.428 & 0.089471 & 0.766091 \\
\hline SD & 227.207 & 61.5974 & 3.688586 & 0.060498 \\
\hline PS & 0.3141 & 100.013 & 0.003141 & 0.955532 \\
\hline AA & 48.3706 & 52.6491 & 0.918736 & 0.342419 \\
\hline SB & 103.934 & 129.286 & 0.803912 & 0.374223 \\
\hline RE & 226.082 & 44.2672 & 5.10722 & 0.028215 \\
\hline SN & 248.271 & 148.219 & 1.675024 & 0.201533 \\
\hline AC & 186.566 & 79.8821 & 2.335521 & 0.132755 \\
\hline TO & 336.944 & 65.8623 & 5.115881 & 0.028088 \\
\hline BI & 143.718 & 49.0072 & 2.9326 & 0.093004 \\
\hline CO & 529.479 & 134.061 & 3.949546 & 0.052376 \\
\hline LC & 1.73815 & 107.24 & 0.016208 & 0.899205 \\
\hline LI & 18.6001 & 89.9265 & 0.206837 & 0.651226 \\
\hline EI & 17.2446 & 120.134 & 0.143545 & 0.706386 \\
\hline SP & 343.441 & 76.1158 & 4.512088 & 0.038625 \\
\hline FX & 464.313 & 82.5149 & 5.627014 & 0.021575 \\
\hline FE & 192.593 & 74.3882 & 2.589023 & 0.113903 \\
\hline
\end{tabular}

\begin{tabular}{|l|c|c|}
\hline \multicolumn{1}{|c|}{ Prueba } & Valor & Nivel-p \\
\hline Lambda de Wilks Rao R Form 2 (18, 33) & $\begin{array}{l}0.404875 \\
2.694818\end{array}$ & 0.006584 \\
\hline \multirow{2}{*}{ Traza de Pillai-Bartlett V (18,33) } & $\begin{array}{l}0.595125 \\
2.694818\end{array}$ & 0.006584 \\
\hline
\end{tabular}

pretest-postest del grupo control (C1-C2) en el total de rasgos proporciona cambios no significativos.

Como se vio en los resultados, entre la primera aplicación del grupo experimental (E1) y la segunda aplicación del grupo control (C2) no existe una diferencia significativa global, lo cual está en la dirección de lo esperado. Sin embargo, a nivel de rasgos particulares se encuentran algunos cambios. Uno de ellos, ya mencionado con anterioridad para el grupo control, es el del rasgo comunalidad que, como también se hipotetizó, puede estar relacionado con variables académicas inherentes a ese grupo ya que en este caso también se ve el cambio en otros rasgos asosiados, como en el caso de responsabilidad y el de sentido psicológico, haciendo este último referencia al interés por las necesidades y motivaciones de los demás, lo cual a su vez ajusta (teóricamente) con el rasgo de feminidad que también cambió significativamente, y con el de comunalidad expresado antes.

En cuanto al análisis realizado entre el postest del grupo experimental (E2) y el pretest del grupo control (C1) observamos que de manera global no presenta diferencia significati- 
va, aunque sí está cercana, y eso puede deberse a que sólo en una pequeña proporción de rasgos -tres de los dieciocho-, se obtiene un cambio significativo, sin embargo, estos tres rasgos están en dirección de lo esperado ya que los tres forman parte de los dos factores involucrados en la autoestima, esto es, la eficacia personal, representada en este caso por el rasgo sociabilidad, el cual, requiere confianza en sí mismo y el factor denominado sentido de mérito personal, representado aquí por los rasgos autoaceptación y sentido de bienestar.

El último análisis en este caso y referido a la comparación entre el postest del grupo experimental (E2) y el postest del grupo control (C2) en cuanto al análisis global, es decir el del conjunto de rasgos resulta como es de esperarse, con diferencias significativas entre ambos grupos. Sin embargo adentrándose en el análisis por rasgo significativo, observamos cómo la diferencia obedece tanto a variables inherentes al grupo experimental como a los factores que influyeron particularmente en el grupo control.

Puede observarse, cómo rasgos que resultaron significativos en la comparación entre el pretest del grupo experimental y el postest del grupo control (E1-C2) resultan también significativos en la comparación presente (E2-C2); estos rasgos son el de responsabilidad y el de sentido psicológico; como ya se comentó anteriormente, este cambio puede estar relacionado con variables académicas inherentes a este grupo control ya que varía con el grupo experimental en sus dos exposiciones (pretest-postest).

Una de las variables en que varía significativamente el grupo experimental del control en la condición postest (E2-C2) es la sociabilidad y la flexibilidad, que en su sentido de adaptabilidad están asociados a la autoestima en cuanto a su competencia y seguridad.

Con base a lo expuesto anteriormente, podemos concluir en términos generales y en dirección de la hipótesis alterna, que los promedios de las puntuaciones obtenidos por los alumnos en el «Inventario de Configuración Psicológica Individual» en aquellos rasgos que están más relacionados con el desarrollo humano y particularmente con la autoestima son significativamente favorables. Por lo tanto, podemos concluir que la aplicación del Programa de Estrategias Metacognitivas para el Desarrollo Humano, dirigido al desarrollo personal de los universitarios de la Facultad de Psicología Veracruzana, produce cambios sustanciales en los rasgos relacionados con dicho desarrollo.

\section{Bibliografía}

Alonso, J. (1994). Motivación y aprendizaje en el aula: Como enseñar a pensar. Madrid: Santillana. Alonso, J. (1992). Leer, comprender y pensar: Nuevas estrategias y técnicas de evaluación. Madrid: C.I.D.E.

Anderson, C. A. y cols. (1994). Atributional style of lonely and depressed people. Journal of Personality and Social Psychology, 45, 127-136.

Arkin, R. y cols. (1988). Social anxiety, self-presentation and the self-serving bias in causal attributions. Journal of Personality and Social Psychology. 38, 23-35.

Ausubel, D. (1980). Psicología de la educación. Un punto de vista cognoscitivo. México: Trillas.

Bandura, A. (1991). Pensamiento y acción. Fundamentos Sociales. Barcelona: Martínez Roca.

Bandura, A. (1989). Percerved self-efficacy in the exercise of personal agency. The Psychologist. 10, 411-424.

Bandura, A. (1984). Teoría del aprendizaje social. Madrid: Espasa-Calpe. 
Bandura, A. (1977). «Self-efficacy: toward a unifing theory of behavioral change». Psychology Reviw, 84, 191-215.

Bandura, A. (1973). Agression: a social learning analysis. Engleed Cliffs, N. J.: Prentice-Hall.

Bandura, A. (1969). Principles of behaviour modification. N.J.: Rinehart and Winston.

Bednar, R. (1991). Self-Esteem: Paradoxes and Innovations in Clinical Theory and Practice. Washington: American Psychology Association.

Belmont, J. M. (1990). Cognitive strategies an strategie learning. American Psychologist, 44, 142148.

Bem, D. (1982). Self-perception theory. En L. Berkowitz (de.), Advances in experimental social psychology. Nueva York: Academic. Vol. 6.

Branden, N. (1996). Los seis pilares de la autoestima. México: Paidós.

Branden, N. (1994). El poder de la autoestima. México: Paidós.

Bruner, J. y Goodman, C. (1947). Value as need as organizing factores in perception. Journal of Abnormal and Social Psychology. 43, 23-44.

Carvallo, R. (2000). Desarrollo Humano a través de Estrategias Metacognitivas: su incidencia en la autoestima. Tesis doctoral. UNED.

Carvallo, R. (1997). Programa de Estrategias Metacognitivas para el Desarrollo Humano. Trabajo Inédito

Coopersmith, S. (1991). The antecedent of self-esteem. San Francisco: Freman.

De Bono, E. (1998). Pensar bien. México: Selector.

De Bono, E. (1994). Saber pensar. México: Selector.

De Sánchez, M. A. (1996). Desarrollo de habilidades del pensamiento: procesos_básicos del pensamiento. México: Trillas.

Dendaluce, I. (1988). Aspectos metodológicos de la investigación educativa. Madrid: Narcea.

Echebarría, A. (Ed.), (1991). Psicología social sociocognitiva. Bilbao: Desclée de Bowner.

Echebarría, A. y Villarreal, M. (1991). La percepción Social. En Echebarría (ed.), Psicología Social Sociocognitiva. Bilbao: DDB.

Eiser, J. R. (1990). Psicología Social: actitudes, cogniciones y conducta social. Madrid: Pirámide.

Erser, J. R. (1983). Attribution theory and social cognition. En J. Jaspars y cols. (eds.), Attribution theory and research: conceptual, developmental and social dimension. Londres: Academic.

Evans, P. (1992). Motivación. México: Continental.

Fiske, S. y Taylor, S. (1994). Social cognition. Nueva York: McGraw-Hill.

Flavell, J. (1985). Speculations about the nature and development of metacognition. En F. Weinert y R. Kluwe (eds.), Metacognition, motivation and understanding. Hillsdale: LEA.

Goleman, D. (1998). Inteligencia emocional. Buenos Aires: Javier Vergara Editor.

Gough, H. G. (1990). Configuración Psicológica Individual. México: Editorial Manual Moderno.

Higgins, E. T. y Bargh, J. A. (1989). Social cognition a social perception. Annual Review of Psychology, 38, 369-425.

Langer, E. (1989). «The psychology of chance». Journal for the Theory of Socialo Behavior, 17, 185208.

León, J. y Gómez, T. (1998). Percepción Social. En León, J. y otros, Psicología Social. España: McGraw-Hill.

León, R. J. (1998). Control psicológico. En León, J. y otros, Psicología_Social. España: McGraw-Hill. 
León, J. y Gómez, T. (1998). Atribución Causal. En León, J. y otros, Psicología_Social. España: McGraw-Hill.

León, J., Medina, S., Barriga, S. y Cantero, F. (1998). Bases Sociales de la emoción. En León, J. y otros, Psicología_Social. España: McGraw-Hill.

Loscertales, F. (1998). Construcción Social de la identidad personal. En León, J. y otros, Psicología Social. España: McGraw-Hill.

Markus, H. y Wurf, E. (1990). The dynamic self-concept: A social psychological perspective. Annual Review of Psychology Bulletin, 8, 189-194.

Mischel, W. (1988). «Metacognition and the rules of delay», en J. Flavell y L. Ross (Eds.), Social Cognitive Development. Cambridge: Cambridge University Press.

Mischel, W. (1986). Introducción a la personalidad. México: Interamericana.

Ornstein, P. (1994). Knowledge and strategies. En W. Schneider y F. Weinert (eds.), Interactions among aptitudes, strategies and knowledge in cognitive performance. Nueva York: Springer.

Paris, S., Wixon, K. y Palinesar, A. (1986). «Instructional approaches to reading comprehension», en E. Rothkopf (Ed.), Review of research in education, vol. 13. Washington: American Educational Research.

Paris, S. y Byrnes, J. (1989). The constructivist approach to self-regulation and learning and academic achievement. Nueva York: Springer.

Pérez Juste, R. (1991). Educación de calidad y calñidad de la educación. Madrid: Antología UNED.

Repetto, E., Téllez, J. A. y Beltrán, S. (2001). Intervención psicopedagógica para la mejora de la comprensión lectora y del aprendizaje. Madrid: UNED.

Repetto, E., Sutil, I., Manzano, N., Beltrán, S. y Téllez, J. (2001). Programa Comprender y Aprender en el Aula. Cuaderno del Alumno: $2^{\circ}$ y $3^{\circ}$ Ciclo de Educación Primaria y $1^{\circ}$ Ciclo de E.S.O. Madrid: Cuadernos de la UNED.

Repetto, E., Sutil, I., Manzano, N., Beltrán, S. y Téllez, J. (2001). Programa Comprender y Aprender en el Aula. Guía del profesor: $2^{\circ}$ y $3^{\circ}$ Ciclo de Educación Primaria y $1^{\circ}$ Ciclo de E.S.O. Madrid: Cuadernos de la UNED.

Repetto, E., Barrero, N. y García, L. (1990). El entrenamiento metacognitivo, la modificabilidad cognitiva y su transferencia a la comprensión lectora, a la resolución de problemas y al aprendizaje. Revista de Investigación Educativa, 8, 563-587.

Ross, L. y Anderson, C. (1992). Shortcomings in the attribution procesess: on the origins and maintenance of erroneous social assessments. En D. Kahneman y cols. (eds.), Judgment under uncertainty: Heuristics and biases. Nueva York: Cambridge University Press.

Schachter, S. y Singer, J. (1962). Cognitive, social and physiological determinants of emotional state. Psychological Review, 69, 379-399.

Schlenker, B. y Wigold, M. (1992). Interpersonal processes involving impression regulation and management. Annual Review of Psychology, 43.

Schunck, D. (1990). Social cognitive theory and self-regulated learning. En B. Zimmerman y D. Schunck (eds.), Self regulated learning and academic achievement. Nueva York: Springer.

Schwartz, G., Shapiro, D. y Davidson, R. (1986). Consciousness and self-regulation. Vol.4, Nueva York: Plenum.

Steele, C. (1995). The psychology of self-affirmation: sustaining the integrity of the self. En Berkowitz (de.), Advances in experimental social psychology, Vol. 21. Orlando, FL: Academic Press.

Taylor, N. (1990). Metacognitive ability: a curriculum priority. Reading Psychology an International Quarterly. 4, 269-278. 
Tice, D. (1991). Esteem protection or enhancement? Self-handicapping motives and attributions differ by trait self-esteem. Journal of Personality and Social Psychology, 60, 711-725.

Wong, B. (1994). The relevance of metacognition to learning disabilities. En B. Wong (ed.), Learning about learning disabilities. San Diego: Academic.

Wong, P. y Weiner, B. (1981). «When people ask "why" questions and the heuristics of attributional research». Journal of Personality and Psychology. 40, 650-663

Wylie, R. C. (1992). The self-concept. Vol. II, Theory and research on selected topics. Lincoln: University of Nebraska Press.

Fecha de recepción: 08/01/03

Fecha de revisión: 26/02/03

Fecha de aceptación: 20/05/03 